\title{
Punishment and psychopathy: A case-control functional MRI investigation of reinforcement learning in violent antisocial personality disordered men
}

ARTICLE in THE LANCET PSYCHIATRY · FEBRUARY 2015

DOI: 10.1016/S2215-0366(14)00071-6

CITATIONS

2

7 AUTHORS, INCLUDING:

Andy Simmons

King's College London

500 PUBLICATIONS $\quad \mathbf{2 0 , 2 5 0}$ CITATIONS

SEE PROFILE

\section{Hodgins Sheilagh}

King's College London

279 PUBLICATIONS 5,966 CITATIONS

SEE PROFILE

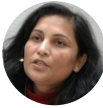

Veena Kumari

King's College London

290 PUBLICATIONS 8,478 CITATIONS

SEE PROFILE

Nigel Blackwood

King's College London

27 PUBLICATIONS 1,382 CITATIONS

SEE PROFILE 


\section{Punishment and psychopathy: a case-control functional MRI investigation of reinforcement learning in violent antisocial personality disordered men}

Sarah Gregory, R James Blair, Dominic ffytche, Andrew Simmons, Veena Kumari, Sheilagh Hodgins, Nigel Blackwood

\section{Summary}

Background Men with antisocial personality disorder show lifelong abnormalities in adaptive decision making guided by the weighing up of reward and punishment information. Among men with antisocial personality disorder, modification of the behaviour of those with additional diagnoses of psychopathy seems particularly resistant to punishment.

Methods We did a case-control functional MRI (fMRI) study in 50 men, of whom 12 were violent offenders with antisocial personality disorder and psychopathy, 20 were violent offenders with antisocial personality disorder but not psychopathy, and 18 were healthy non-offenders. We used fMRI to measure brain activation associated with the representation of punishment or reward information during an event-related probabilistic response-reversal task, assessed with standard general linear-model-based analysis.

Findings Offenders with antisocial personality disorder and psychopathy displayed discrete regions of increased activation in the posterior cingulate cortex and anterior insula in response to punished errors during the task reversal phase, and decreased activation to all correct rewarded responses in the superior temporal cortex. This finding was in contrast to results for offenders without psychopathy and healthy non-offenders.

Interpretation Punishment prediction error signalling in offenders with antisocial personality disorder and psychopathy was highly atypical. This finding challenges the widely held view that such men are simply characterised by diminished neural sensitivity to punishment. Instead, this finding indicates altered organisation of the informationprocessing system responsible for reinforcement learning and appropriate decision making. This difference between violent offenders with antisocial personality disorder with and without psychopathy has implications for the causes of these disorders and for treatment approaches.

Funding National Forensic Mental Health Research and Development Programme, UK Ministry of Justice, Psychiatry Research Trust, NIHR Biomedical Research Centre.

\section{Introduction}

Most violent crimes are committed by a small number of men. ${ }^{1}$ They display a pattern of antisocial and aggressive behaviour that begins in childhood and remains stable throughout the lifespan. They meet diagnostic criteria for conduct disorder in childhood and for antisocial personality disorder in adulthood. Lifelong patterns of poor decision making, impulsivity, and risk-taking behaviours characterise such men, and persist despite repeated punishments enacted by parents, teachers, and the criminal justice system.

Within this population, a subgroup presents with antisocial personality disorder and psychopathy, defined by the psychopathy checklist-revised (PCL-R) ${ }^{2}$ as including callousness, lack of empathy, an interpersonal style involving grandiosity and manipulation of others, and persistent reactive and instrumental aggression. Such individuals show reduced tonic skin conductance and cortisol concentrations, and have difficulty recognising fear and sadness in the faces of others. ${ }^{3}$ By contrast, men who have antisocial personality disorder without psychopathy are characterised by emotional lability, mood and anxiety disorders, and reactive aggression. ${ }^{4}$ Although the two phenotypes emerge early in life, those who develop antisocial personality disorder with psychopathy begin offending at an earlier age, engage in a broader range and higher frequency of offending behaviours, ${ }^{4}$ and respond less well to treatment in childhood ${ }^{5}$ and adulthood ${ }^{6}$ than those without psychopathy. The two groups show distinct differences in brain structure ${ }^{7}$ and functional responses to empathy-eliciting scenarios ${ }^{8}$ and emotional stimuli when engaged in goal-directed behaviour.' Diagnostic classification systems, however, do not distinguish between antisocial personality disorder with and without psychopathy. Rehabilitation programmes typically exclude offenders with psychopathy.

Many decisions, including whether or not to engage in an antisocial act, involve the ability to assess consequences. Selecting an action is dependent on reinforcement learning, whereby possible rewards are weighed against possible punishments on the basis of past experience. ${ }^{10}$ Men with antisocial personality disorder show impairments in adaptive decision making, characterised by abnormal processing of reinforcement information.1,12 ${ }^{112}$ Their
Lancet Psychiatry 2015; 2: $153-60$

See Comment page 115

Department of Forensic and Neurodevelopmental Sciences, Institute of Psychiatry, King's College London, London, UK (S Gregory PhD, N Blackwood MD); Mood and Anxiety Disorders Program, National Institute of Mental Health, National Institutes of Health, Bethesda, MD, USA (Prof RJ Blair PhD); Department of Old Age Psychiatry, Institute of Psychiatry, King's College London, London, UK (D ffytche MD); NIHR Biomedical Research Centre for Mental Health at South London and Maudsley NHS Foundation Trust and Institute of Psychiatry, King's College London, London, UK (A Simmons PhD, Prof V Kumari PhD); and Département de Psychiatrie, Université de Montréal, Montréal, QC, Canada (Prof S Hodgins PhD) Correspondence to: Dr Nigel Blackwood, Department of Forensic and Neurodevelopmental Sciences, Institute of Psychiatry, Kings College London, PO Box 23, De Crespigny Park, London SE5 8AF, UK nigel.blackwood@kcl.ac.uk 
behaviour seems to be driven more by potential rewards than potential punishments (reward dominance). Impaired learning about the consequences of actions might result in overly optimistic and inaccurate predictions of favourable outcomes, resulting in frustration, which could predispose to reactive aggression. Impaired self-control lowers the threshold for aggression in response to such frustration. ${ }^{13}$ In behavioural studies offenders with antisocial personality disorder and psychopathy have shown notable impairment in using reinforcement information when choosing between punished and rewarded objects in passive avoidance ${ }^{14}$ extinction, ${ }^{15}$ and reversal learning tasks. ${ }^{11,16,17}$ The failure to use punishment information to signal inappropriate behaviour is thought to be the primary deficit in psychopathy, and emerges early in childhood. ${ }^{18}$

Reversal learning tasks examine the ability to adjust behaviour to changes in reinforcement contingency. Studies of healthy adults and adults with brain lesions have identified a neural network recruited during reversal learning. ${ }^{19}$ The dorsomedial prefrontal cortex, ventrolateral prefrontal cortex, and dorsolateral prefrontal cortex serve, respectively, to allocate attentional resources, assess conflicting responses, and initiate response modulation. The ventromedial prefrontal cortex, posterior cingulate cortex, and dorsal caudate track changes in reinforcement information, reducing activation in response to punishment, thus signalling behavioural error, change in reinforcement contingency (when reward is expected but punishment received), and the need to adapt behaviour. ${ }^{20,21}$

Little is known about the neural systems serving reinforcement learning anomalies in offenders with antisocial personality disorder with and without psychopathy. Offline versions of probabilistic response reversal tasks are self-paced, which means that intervals between the stimulus presentation, response, and feedback vary across trials. ${ }^{11} \mathrm{~A}$ variant of this task has been developed for the functional MRI (fMRI) scanning environment that is designed specifically to minimise behavioural differences between antisocial individuals and normal controls. Importantly, the scanner version of the task provides reinforcement more often and more consistently than the offline task. This change lessens task difficulty and the likelihood of confounding introduced by large differences in performance, such as differential contamination by error events. ${ }^{22}$ The scanner version of the task has been used to assess adolescents with conduct disorder and callous unemotional traits, ${ }^{21}$ which are the childhood antecedents of antisocial personality disorder with psychopathy. ${ }^{5}$ Compared with healthy teenagers, adolescents who have conduct disorder with callous unemotional traits showed intact recruitment of the dorsomedial prefrontal cortex, dorsolateral prefrontal cortex, and inferior frontal cortices when changing their behaviour in response to punishment signals, but did not appropriately reduce activation of the ventromedial prefrontal cortex. This finding was interpreted as intact recruitment of regions involved in response change immediately after punishment, but impairment in regions that alter longterm value associated with responses, thereby increasing the likelihood of future incorrect responding.

We used the scanning environment probabilistic response reversal task to do an fMRI study of reinforcement processing and decision making in violent adult offenders with antisocial personality disorder with and without psychopathy. Focusing on responses to punished reversal errors relative to rewarded correct responses, we tested the hypothesis that violent offenders with antisocial personality disorder and psychopathy would show increased activation within the ventromedial prefrontal cortex, caudate, and posterior cingulate cortex to punished reversal errors, as identification of anomalous responding to reinforcement information within such individuals could be useful as a diagnostic biomarker.

\section{Materials and methods Participants and study design}

Between January, 2007, and January, 2011, we enrolled 50 men, aged 20-50 years, with reading age higher than 10 years as defined by the Schonell instrument. Eligible participants had no history of major mental disorders (bipolar 1, bipolar 2, major depression, or psychotic disorders), as defined by the structured clinical interview for Diagnostic and Statistical Manual of Mental Disorder, fourth edition (DSM-IV), axis I disorders (SCID I), or selfreported neurological disorders, head injury resulting in loss of consciousness for $1 \mathrm{~h}$ or longer, severe visual or hearing impairments, or contraindications to MRI Offenders with convictions for violent crimes (murder, rape, attempted murder, and grievous bodily harm) who met DSM-IV criteria for antisocial personality disorder were recruited via the National Probation Service of England and Wales. Healthy non-offenders were recruited via community websites and from unemployment offices. All participants completed diagnostic and PCL-R interviews and authorised access to their criminal records. A cross-cultural validation study $^{23}$ of the PCL-R demonstrated that cutoff scores for psychopathy in men vary between North America (30 of a possible 40 points) and Europe ( 25 of a possible 40 points). We used a score of 25 as the threshold for psychopathy. We calculated total PCL-R, factor 1 (interpersonal and affective traits) and factor 2 (impulsivity and antisocial lifestyle) scores for all participants.?

This study was approved by the Joint South London and Maudsley and the Institute of Psychiatry NHS Research and Ethics Committee. Participants were paid the UK minimum hourly wage for their time and were encouraged to desist from using substances, including alcohol and illicit drugs (appendix), from 2 weeks before and during the study, and use was assessed in urine and saliva samples collected at each session.

\section{Measures}

Forensic psychiatrists did diagnostic interviews in which they assessed participants with SCID I and SCID for axis 
II disorders (SCID II) in video-taped interviews. Trained forensic psychiatrists and psychologists administered the PCL-R in interviews, whichwere also video-taped. A random sample of $25 \%$ of participants was rerated by a second psychologist to test the intraclass correlation for total scores. All participants completed the Wechsler adult intelligence scale, third edition, and the reactiveproactive aggression questionnaire.

\section{Probabilistic response reversal task}

The scanning environment event-related probabilistic response reversal task assesses the ability to learn (acquisition phase) and alter (reversal phase) stimulusresponse associations as a function of contingency change. Participants were presented with pairs of images (line drawings of animals or furniture) on a screen while in the fMRI scanner and asked to select one. Each choice received positive or negative feedback (gain or loss of points; figure 1). To increase task difficulty, stimulus pairs had reinforcement probabilities of 100:0 (positive or negative feedback was given for $100 \%$ of right or wrong selections, respectively) or 80:20 (positive or negative feedback was given for $80 \%$ of right or wrong selections, respectively, but swapped for $20 \%$ of trials). In the acquisition phase, pairs of images were presented 20 times for the 100:0 condition or 25 times for the 80:20 condition, with each individual image assigned rewarding reinforcement. In the reversal phase, the reinforcement contingency associated with the pair of images then reversed and they were presented a further 20 or 25 times. Running point totals were displayed for each acquisition or reversal trial (appendix).

\section{Image acquisition and processing}

Participants were scanned in a 1.5 T Excite MRI scanner (GE Medical Systems, Hatfield, UK), with use of an eightchannel head coil. Four dummy acquisition scans were

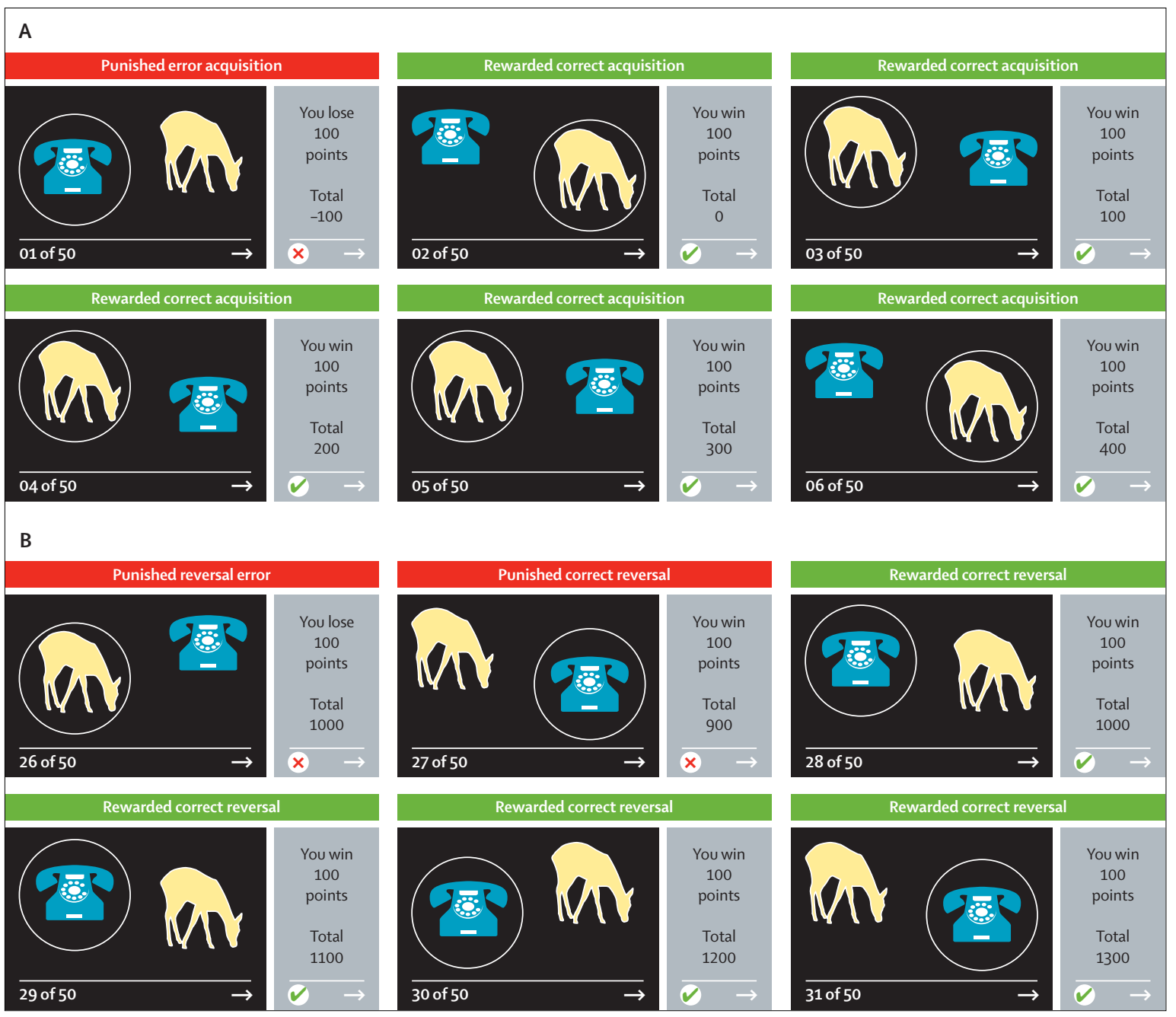

Figure 1: Schematic of the probabilistic response-reversal task

(A) The acquisition phase. (B) The reversal phase. 80:20 reinforcement probability is illustrated, where positive or negative feedback was given for $80 \%$ of right or wrong selections, respectively, but was swapped for $20 \%$ of trials. Participants selected one of the pair of images and received rewarding or punishing feedback for 25 acquisition trials, after which the contingency was reversed for 25 trials so that previously rewarded images resulted in punishment. 
For the SPM toolbox see http:// marsbar.sourceforge.net// used to establish steady-state longitudinal magnetisation. For each participant, $192 \mathrm{~T}_{2}{ }^{*}$-weighted images were acquired with a gradient-echo, planar, imaging sequence with repetition time $2500 \mathrm{~ms}$, echo time $40 \mathrm{~ms}$, flip angle $90^{\circ}$, and in-plane resolution $3.75 \mathrm{~mm}^{2} .25$ axial slices of $5 \mathrm{~mm}$ thickness with $0.5 \mathrm{~mm}$ gaps between were collected. A high-resolution T1-weighted spoiled gradient re-echo image was also acquired, which was used for coregistration and normalisation of the data. 124 slices of $1.6 \mathrm{~mm}$ thickness were collected with repetition time $34 \mathrm{~ms}$, echo time $9 \mathrm{~ms}$, flip angle $30^{\circ}$, field of view $20 \mathrm{~cm}$, and an acquisition matrix of $256 \times 192$.

\section{Statistical analysis}

Demographic and clinical variables were compared with two-sample $t$ tests, ANOVA, or $\chi^{2}$ and Fisher's exact tests. To test for differences in performance between groups, the percentage of errors and response latency were each assessed with $3 \times 2$ (group $\times$ phase) repeated-measures ANOVA. In the general linear model, we applied seven conditions modelled at the first level for each participant: baseline; rewarded correct acquisition; rewarded correct reversal; punished correct acquisition; punished correct reversal; punished errors acquisition; punished errors reversal. The numbers of trials within each of these conditions (excluding the motor baseline which was consistent for each block) for each group were also assessed with one-way ANOVA. All analyses were done with SPSS (version 15).

Image preprocessing and analyses were done with SPM5 software (running under Matlab 7.0.1 on a UNIX platform). All fMRI time series were realigned, coregistered to the spoiled gradient re-echo image, spatially normalised to the International Consortium for Brain Mapping template image and smoothed with an $8 \mathrm{~mm}$ full width at half maximum Gaussian kernel. Movement parameters were included as regressors. At the group level we assessed the contrast in blood oxygenation-level-dependent (BOLD) responses for punished reversal errors versus rewarded correct responses (rewarded correct acquisition + rewarded correct reversal).

One-sample $t$ tests were done to identify punished reversal error and rewarded correct response task systems for each group, with an initial significance threshold of $\mathrm{p}=0.001$ and a familywise error cluster corrected significance threshold of $0 \cdot 05$. The $t$ test values, which were modelled within the SPM factorial design, were calculated to investigate potential differences between groups to the punished reversal error and rewarded correct response trials, with an initial significance threshold of $\mathrm{p}=0.005$ and familywise error cluster corrected. We extracted $\beta$ values from regions of differential activation from men with antisocial personality disorder with and without psychopathy by use of the SPM toolbox for illustrative purposes. Finally, a secondary analysis was done to explore whether the observed group differences in BOLD contrast between offenders with antisocial personality disorder with and without psychopathy for punished reversal errors versus rewarded correct responses were specifically related to the syndrome of psychopathy or could alternatively be explained by antisocial personality disorder symptom severity. Thus, independent correlations between activity during punished reversal errors compared

\begin{tabular}{|c|c|c|c|c|c|c|c|c|}
\hline & \multicolumn{3}{|l|}{ Group } & \multicolumn{2}{|l|}{ Group comparison } & \multicolumn{3}{|l|}{ Post-hoc test } \\
\hline & NO $(n=18)$ & ASPD-P $(n=20)$ & ASPD $+P(n=12)$ & Statistic & p value & NO vs ASPD-P & $\mathrm{NO}$ us ASPD+P & ASPD-P vs ASPD+P \\
\hline Age (years) & $34.8(8.8)$ & $36 \cdot 8(7 \cdot 6)$ & $40 \cdot 1(8 \cdot 9)$ & $\mathrm{F}_{2,47}=1 \cdot 39$ & 0.26 & 1 & 0.31 & 0.88 \\
\hline FSIQ score & $97 \cdot 2(11 \cdot 0)$ & $91 \cdot 7(12 \cdot 2)$ & $89 \cdot 8(11 \cdot 2)$ & $\mathrm{F}_{2,47}=1.71$ & 0.19 & 0.46 & $0 \cdot 3$ & 1 \\
\hline Duration of education (years) & $12 \cdot 2(1 \cdot 5)$ & $10 \cdot 5(0 \cdot 8)$ & $10 \cdot 4(1 \cdot 2)$ & $F_{2,47}=11.43$ & 0.001 & 0.001 & 0.001 & 1 \\
\hline \multicolumn{9}{|l|}{ Personality disorder other than ASPD (\%) } \\
\hline Cluster A & 0 & 10 & $8 \cdot 3$ & 1.92 & 0.45 &.. & .. &.. \\
\hline Cluster B & 0 & 10 & $8 \cdot 3$ & 1.92 & 0.45 &.. & .. &.. \\
\hline Cluster C & 0 & 5 & $8 \cdot 3$ & 1.65 & 0.71 &.. &.$\cdot$ &.. \\
\hline \multicolumn{9}{|l|}{ PCL-R score (mean [range]) } \\
\hline Total $(0-40)$ & $3 \cdot 4(0-8)$ & $15 \cdot 9(10-24)$ & $28 \cdot 2(26-30)$ & $F_{2,45}=246 \cdot 79$ & 0.001 & 0.001 & 0.001 & 0.001 \\
\hline Factor $1(0-20)$ & $0.6(0-4)$ & $4 \cdot 5(0-11)$ & $9 \cdot 6(6-13)$ & $F_{2,45}=55 \cdot 36$ & 0.001 & 0.001 & 0.001 & 0.001 \\
\hline Factor $2(0-20)$ & $2 \cdot 3(0-6)$ & $10 \cdot 3(5-15)$ & $15 \cdot 8(12-19)$ & $F_{2,45}=110 \cdot 70$ & 0.001 & 0.001 & 0.001 & 0.001 \\
\hline Age at first violent conviction (years) & $\mathrm{N} / \mathrm{A}$ & $22 \cdot 3(9 \cdot 9)$ & $17 \cdot 1(3 \cdot 8)$ & $\mathrm{t}=2 \cdot 08, \mathrm{df}=27$ & 0.05 &.. &.$\cdot$ &.. \\
\hline Number of violent convictions & $\mathrm{N} / \mathrm{A}$ & $4 \cdot 5(3 \cdot 2)$ & $7 \cdot 6(5 \cdot 9)$ & $\mathrm{t}=-1 \cdot 68, \mathrm{df}=15$ & 0.11 &.. & .. &.. \\
\hline \multicolumn{9}{|l|}{ Aggression score } \\
\hline Total & $7 \cdot 1(3 \cdot 6)$ & $15 \cdot 7(7 \cdot 3)$ & $22 \cdot 9(12 \cdot 0)$ & $F_{2,42}=13 \cdot 44$ & 0.001 & 0.001 & 0.002 & 0.22 \\
\hline Proactive & $2 \cdot 0(2 \cdot 3)$ & $7 \cdot 4(3 \cdot 9)$ & $13 \cdot 8(6 \cdot 6)$ & $F_{2,42}=24.03$ & 0.012 & 0.001 & 0.001 & 0.02 \\
\hline Reactive & $5 \cdot 1(3 \cdot 4)$ & $8 \cdot 3(5 \cdot 7)$ & $11 \cdot 7(7 \cdot 0)$ & $F_{2,42}=4.91$ & 0.001 & 0.151 & 0.027 & 0.44 \\
\hline
\end{tabular}

Group data are mean (SD) unless stated otherwise. NO=non-offenders. ASPD-P=violent offenders with antisocial personality disorder but not psychopathy. ASPD+P=violent offenders with antisocial personality disorder and psychopathy. FSIQ=full-scale intelligence quotient. PCL-R=psychopathy checklist-revised. N/A=not applicable.

Table 1: Sociodemographic, clinical, and behavioural characteristics of participants 
with rewarded correct responses for PCL-R scores and total number of SCID II antisocial personality disorder symptoms in the entire group of offenders with antisocial personality disorder were modelled.

\section{Role of the funding source}

The funder of the study had no role in the study design, data collection, data analysis, data interpretation, or writing of the report. The corresponding author had full access to all the data in the study and had final responsibility for the decision to submit for publication.

\section{Results}

The three groups did not differ significantly except for education and PCL-R score (table 1). The offenders with antisocial personality disorder and psychopathy were significantly younger at first conviction than those without psychopathy. They also had higher numbers of convictions for violent crime, but this difference was not significant. Significantly more offenders had lifetime diagnoses of alcohol and cocaine dependence than nonoffenders (appendix). Of note, though, the proportions of offenders with and without psychopathy and with lifetime substance use disorders did not differ.

Intraclass correlation for PCL-R total scores was $0 \cdot 83$. Groups did not differ significantly for percentage errors $\left(\mathrm{F}_{2,4}=1 \cdot 22, \mathrm{p}=0 \cdot 31\right)$ or response latencies $\left(\mathrm{F}_{2,47}=0 \cdot 87\right.$, $\mathrm{p}=0.43$ ) in the acquisition and reversal phases of the task (appendix). For percentage errors, there was an effect of phase $\left(\mathrm{F}_{1,47}=107 \cdot 4, \mathrm{p}=0 \cdot 001\right)$, with more errors being committed during the reversal phase, but no significant phase-group interaction $\left(\mathrm{F}_{2,47}=0 \cdot 511, \mathrm{p}=0 \cdot 60\right)$. For response latencies, an effect of phase was seen but was not quite significant $\left(\mathrm{F}_{1,47}=3 \cdot 51, \mathrm{p}=0 \cdot 07\right)$, with responses being slower during the reversal phase, but no significant interaction was seen for phase and group $\left(\mathrm{F}_{2,47}=0 \cdot 15\right.$, $\mathrm{p}=0 \cdot 86$ ). The number of trials for each condition (appendix) and the proportion of trials completed were similar in all groups $(\mathrm{p}=0 \cdot 25)$.

Offenders with antisocial personality disorder and psychopathy, compared with those without psychopathy and non-offenders, displayed increased BOLD responses to punished reversal error trials within the bilateral posterior cingulate cortex and precuneus (Brodmann areas 23 and 31). Increased activation was also seen in offenders with psychopathy during punished reversal errors trials in the right anterior insula when compared with offenders without psychopathy, but not non-offenders. A difference between offenders with antisocial personality disorder and psychopathy and non-offenders, however, did become evident when a lower cluster-defining threshold of $\mathrm{p}=0 \cdot 01$ was used. Conversely, the antisocial offenders without psychopathy and the non-offenders showed significantly increased BOLD responses during rewarded correct response trials in the right superior temporal gyrus (Brodmann area 22), extending to the anterior middle temporal gyrus (Brodmann area 21) compared with

\begin{tabular}{|c|c|c|c|c|c|c|c|}
\hline & \multirow[t]{2}{*}{ Brodmann area } & \multicolumn{3}{|c|}{ MNI coordinates } & \multirow[t]{2}{*}{ Cluster size } & \multirow[t]{2}{*}{ Z score } & \multirow[t]{2}{*}{ p value* } \\
\hline & & $\mathrm{x}$ & y & z & & & \\
\hline \multicolumn{8}{|c|}{ ASPD+P>ASPD-P, punished reversal errors >rewarded correct responses } \\
\hline \multicolumn{8}{|c|}{ Left posterior cingulate cortex } \\
\hline Posterior cingulate cortex & 23 & -3 & -24 & 30 & 818 & $3 \cdot 84$ & 0.001 \\
\hline Posterior cingulate cortex & 31 & 0 & -39 & 27 & .. &.$\cdot$ & \\
\hline Precuneus & 7 & -3 & -69 & 42 & .. &. & \\
\hline \multicolumn{8}{|l|}{ Right posterior cingulate cortex } \\
\hline Posterior cingulate cortex & 31 & 6 & -45 & 36 &. &.$\cdot$ & \\
\hline Precuneus & 7 & 0 & -63 & 36 & 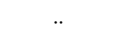 &. & \\
\hline Posterior cingulate cortex & 23 & 9 & -57 & 12 &.. &.. & \\
\hline \multicolumn{8}{|l|}{ Right anterior insula } \\
\hline Inferior frontal gyrus & 44 & 51 & 3 & 18 & 204 & $3 \cdot 16$ & 0.044 \\
\hline Insula &.$\cdot$ & 36 & 3 & 0 & $\cdot \cdot$ &.$\cdot$ & \\
\hline Insula & .. & 33 & 9 & -3 & .. & .. & \\
\hline \multicolumn{8}{|c|}{ ASPD+P>non-offenders, punished reversal errors $>$ rewarded correct responses } \\
\hline \multicolumn{8}{|c|}{ Left posterior cingulate cortex } \\
\hline Posterior cingulate cortex & 23 & -3 & -39 & 27 & 281 & $3 \cdot 47$ & 0.011 \\
\hline Posterior cingulate cortex & 31 & -3 & -63 & 15 &.. &.. & \\
\hline Posterior cingulate cortex & 23 & -6 & -54 & 9 & .. &. & \\
\hline \multicolumn{8}{|l|}{ Right posterior cingulate cortex } \\
\hline Posterior cingulate cortex & 23 & 3 & -54 & 9 &.. &.$\cdot$ & \\
\hline Precuneus & 7 & 3 & -66 & 33 & .. & .. & \\
\hline Precuneus & 7 & 3 & -54 & 39 &.. & .. & \\
\hline
\end{tabular}

$A S P D-P>A S P D+P$, rewarded correct responses $>$ punished reversal errors

Right superior temporal gyrus

$\begin{array}{llllllll}\text { Middle temporal gyrus } & 21 & 54 & -36 & -6 & 211 & 3.68 & 0.039\end{array}$

Superior temporal gyrus

Superior temporal gyrus

21

$60-18 \quad-3$

Non-offenders $>A S P D+P$, rewarded correct responses $>$ punished reversal errors

Right superior temporal gyrus

$\begin{array}{lcrrrrrr}\text { Superior temporal gyrus } & 22 & 45 & -18 & 3 & 251 & 3.76 & 0.019 \\ \text { Superior temporal gyrus } & . . & 48 & -6 & 0 & . . & . . & \\ \text { Superior temporal gyrus } & . . & 39 & -15 & 18 & . . & . . & \end{array}$

$\mathrm{BOLD}=$ blood-oxygenation-level-dependent. ASPD- $\mathrm{P}=$ violent offenders with antisocial personality disorder but not psychopathy. ASPD+P=violent offenders with antisocial personality disorder and psychopathy. ${ }^{*}$ Corrected for familywise error.

Table 2: Areas with significantly different BOLD responses for the contrast punished reversal error trials versus rewarded correct response trials

offenders with antisocial personality disorder and psychopathy. No significant differences in BOLD activity in either direction were seen between the offenders with antisocial personality disorder but not psychopathy and non-offenders (table 2, figures 2, 3).

All three groups showed significantly greater activation during punished reversal error trials than during rewarded correct response trials within the ventrolateral prefrontal cortex, dorsolateral prefrontal cortex, and dorsomedial prefrontal cortex. Offenders with antisocial personality disorder with psychopathy and non-offenders also showed significantly greater activity within the inferior parietal lobe during punished reversal error trials than during rewarded correct response trials. Only offenders with antisocial personality disorder and psychopathy showed significantly 


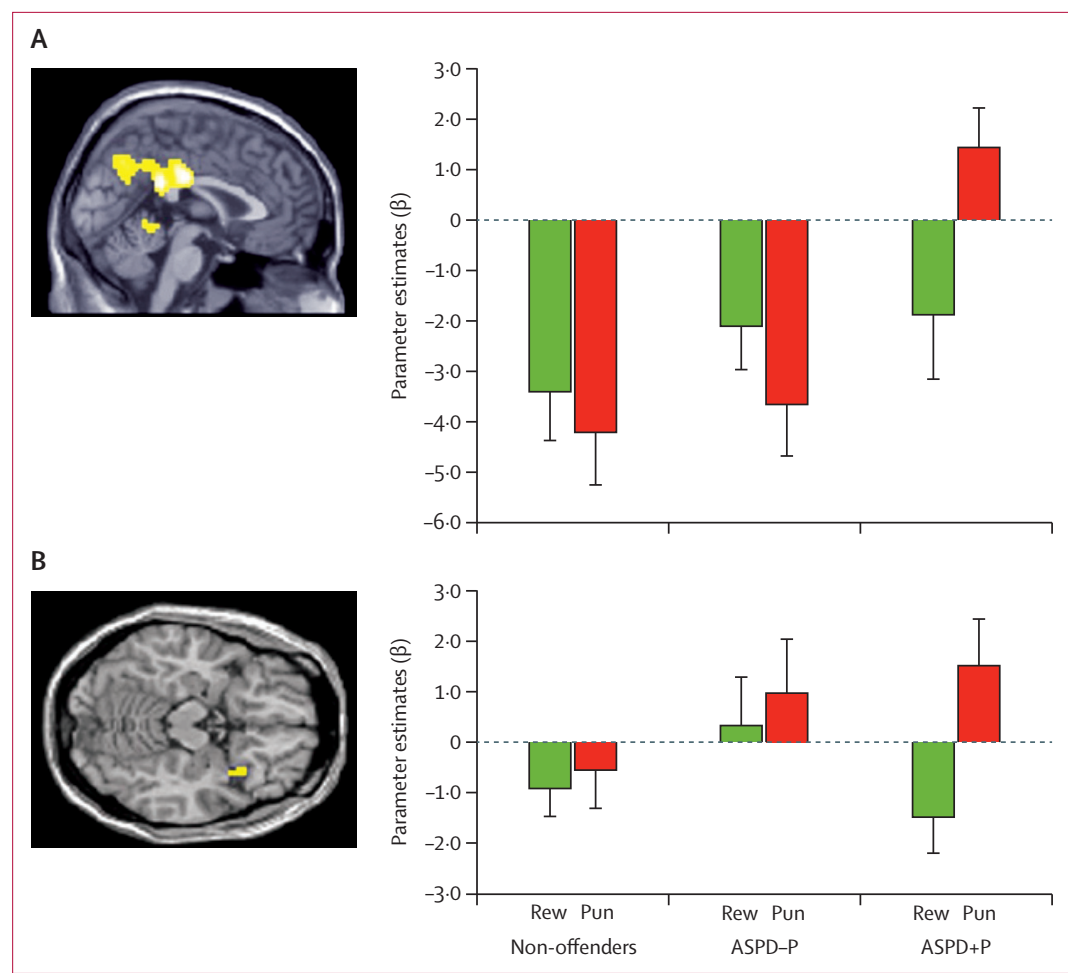

Figure 2: Mean values for the contrast punished reversal errors>rewarded correct responses (A) Bilateral posterior cingulate cortex. (B) Right anterior insula. In these two regions the ASPD+P group showed significantly increased activation to punished reversal errors relative to the two comparison groups. Areas of signficantly increased activation in ASPD+P group compared with the ASPD-P group are overlaid on the MNIT1 template. Error bars represent $\mathrm{SE}$. Rew=rewarded correct responses. $\mathrm{Pun}=$ punished reversal errors. ASPD-P=antisocial personality disorder without psychopathy. $\mathrm{ASPD}+\mathrm{P}=$ antisocial personality disorder with psychopathy.
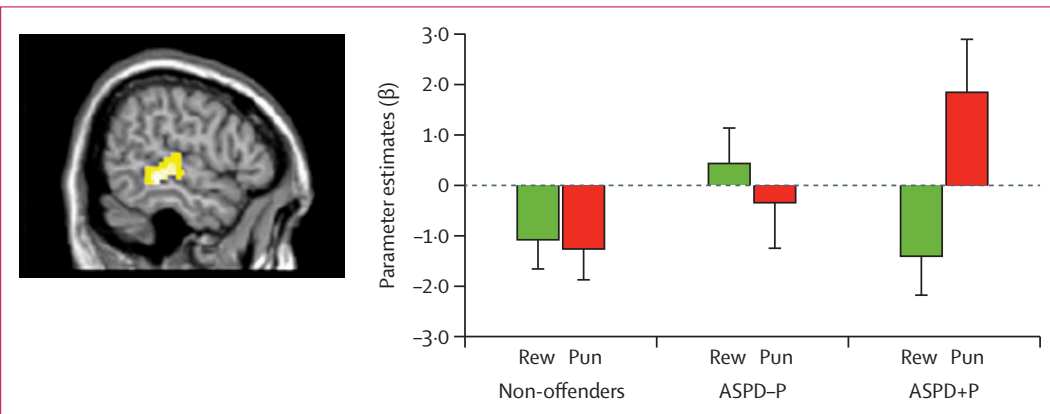

Figure 3: Mean values for the contrast rewarded correct responses>punished reversal errors in the right superior temporal gyrus

In this region the ASPD-P and the non-offender groups showed significantly increased activation to rewarded correct responses relative to the ASPD+P group. Error bars represent $S E$. Rew=rewarded correct responses. Pun=punished reversal errors. ASPD-P=antisocial personality disorder without psychopathy. $A S P D+P=$ antisocial personality disorder with psychopathy.

greater BOLD responses in the posterior cingulate cortex during punished reversal error trials than during rewarded correct response trials. By contrast, those without psychopathy and non-offenders showed significantly greater activity to rewarded correct response trials than to punished reversal error trials within this region and in the superior temporal gyrus. In offenders with antisocial personality disorder and psychopathy, no regions showed significantly greater activity to rewarded correct response trials than to punished reversal error trials. The results remained unchanged when group comparisons were repeated excluding offenders who had positive urinary drug screens on the day of scanning (appendix).

The secondary correlation analyses in offenders showed a positive relation between PCL-R scores and activity within the posterior cingulate cortex region (MNI coordinates $\mathrm{x}=-$ 3 , $y=-66$, and $z=42$, cluster size $323, Z=3 \cdot 52, p=0 \cdot 005$ corrected) during punished reversal error trials versus rewarded correct response trials. However, no significant relation was seen between the number of SCID II antisocial personality disorder symptoms and activation in this area.

\section{Discussion}

We investigated the neural basis of reversal learning in violent offenders with antisocial personality disorder with and without psychopathy. Offenders with antisocial personality disorder and psychopathy displayed abnormal responding to punishment signals within the posterior cingulate and insula, illustrated by significantly greater BOLD activation to punished reversal errors than to rewarded correct responses in both regions. These differences were not related to the number of antisocial personality disorder symptoms. Offenders with antisocial personality disorder but not psychopathy and nonoffenders showed significant reductions in activation in the posterior cingulate cortex, and the offenders without psychopathy had significantly reduced activation in the anterior insula.

Reversal learning impairments have been robustly identified in children with conduct disorder and callous unemotional traits and in adult men with psychopathy. ${ }^{12,24}$ In this study, however, we noted no behavioural deficits, which is consistent with results of the fMRI investigation with the same task in adolescents with conduct disorder and callous unemotional traits. ${ }^{21}$ In these two fMRI studies, abnormal activations were seen in brain regions signalling prediction errors. Adolescents with conduct disorder and callous unemotional traits showed greater activity within the ventromedial prefrontal cortex to punished reversal errors than did healthy adolescent controls. ${ }^{21}$ Offenders with antisocial personality disorder and psychopathy, unlike those without psychopathy and non-offenders, showed significantly greater activation within the posterior cingulate cortex to punished reversal errors. Consistent with previous findings in healthy adults, reduced BOLD responses within the posterior cingulate to punished reversal errors compared with those for rewarded correct responses were seen in the offenders with antisocial personality disorder but not psychopathy and in the non-offenders. ${ }^{19,20}$

The posterior cingulate cortex plays an important part in altering behaviour in response to an unexpected change. ${ }^{25}$ Animal and human studies have shown the relevance of this cortex in representing subjective value, particularly in relation to rewards, ${ }^{26}$ and in error signalling after the omission of an expected reward. ${ }^{27}$ The posterior cingulate 
typically responds to prediction error in conjunction with the ventromedial prefrontal cortex..$^{27}$ In previous studies, structural posterior cingulate cortex abnormalities were seen in children with callous unemotional traits ${ }^{28}$ and some adults with psychopathy, ${ }^{29}$ but these were not seen in the sample in this study. ${ }^{7}$ Reduced posterior cingulate cortex responsivity to emotional stimuli, ${ }^{30}$ including personal moral dilemmas, ${ }^{31}$ has also been seen in investigations of adults with psychopathy. A white-matter diffusion tensor imaging study of the offenders with antisocial personality disorder and psychopathy from this study revealed reduced fractional anisotropy suggestive of reduced axonal integrity and organisation in the dorsal cingulum, which links the posterior cingulate cortex to the medial prefrontal cortex. ${ }^{32}$ The findings from this study support the proposition that the representation of reinforcement value is profoundly disturbed in adult men with psychopathy (panel). ${ }^{33}$

Thus, in adolescents ${ }^{21}$ and adults, rather than psychpathic traits being associated with decreases in activity within reinforcement-sensitive regions after unexpected punishment, significantly greater activation within these regions was seen in response to punished trials. This result challenges the notion that individuals with psychopathy are simply insensitive to subjective value, prediction errors, or both. ${ }^{33}$ Men with psychopathy were sensitive to this information and used it to inform appropriate behavioural change, but processed it in a highly atypical way. This atypical processing was associated with psychopathy but not with the number of antisocial personality disorder symptoms. Replications and further elucidation of this anomalous responding are urgently needed, not least because treatments for psychopathy that increase sensitivity to reinforcement information might be misdirected. Instead, interventions aimed at modifying the subjective value accorded to punishment information, perhaps by focusing attention appropriately, might need to be developed. ${ }^{15}$

Among the offenders with antisocial personality disorder and psychopathy, a similar abnormality was seen within the anterior insula. The anterior insula is connected to limbic regions, such as the ventromedial prefrontal cortex and amygdala, and is innervated by dopaminergic neurons. This area is involved in motivation and represents context-dependent aversive value and reward and tracks the salience of outcomes, including recognition of errors. ${ }^{34}$ Insular damage in human beings promotes risky decision-making because of impaired signalling of the probability of aversive outcomes. ${ }^{35}$ Reductions in grey-matter volume in the insula have been reported in adults with psychopathy, 7,29 as have reductions in insula activity during aversive conditioning $^{30}$ and atypically increased activation in response to empathy-eliciting scenarios. ${ }^{8}$

Regions involved in representing subjective value, such as the ventromedial prefrontal cortex and posterior cingulate cortex, have been proposed to integrate input from the superior temporal gyrus. ${ }^{36}$ In this study, offenders
Panel: Research in context

\section{Systematic review}

We searched PubMed for reports published in English up to Aug 8, 2014, with the search terms "punishment AND psychopathy", "fMRI AND psychopathy", "response reversal AND psychopathy", "reinforcement learning AND psychopathy", and "fMRI AND antisocial". We also checked the reference lists of identified reports for relevant publications. We found no studies that had assessed the neural underpinnings of reversal learning in men with antisocial personality disorder with and without psychopathy.

\section{Interpretation}

We found distinctive neural mechanisms related to the severe impairment in learning from punishment that characterises violent offenders with antisocial personality disorder and psychopathy. Important neurobiological distinctions between men with antisocial personality disorder with and without psychopathy have been theorised. The lack of evidence for such differences, however, has led to diagnostic classification schemes that favour a single diagnosis of antisocial personality disorder, which has greatly hindered progress in understanding the causes of subtypes and their optimum treatment. Child diagnostic classification systems specify the developmental precursor of psychopathy, limited prosocial emotions, as an important subgroup within those with conduct disorder. Our findings add to the weight of evidence encouraging similar specifications in adult diagnostic schemes to distinguish the syndrome of psychopathy from antisocial personality disorder and to take the subgroups into account when planning rehabilitation programmes. As most violent crimes are committed by men with this early-onset stable pattern of antisocial and aggressive behaviour, interventions that target the specific underlying brain mechanisms and effect change in the behaviour have the potential to significantly reduce the rate of violent crime.

with antisocial personality disorder without psychopathy and non-offenders showed significantly greater activity within this region to rewarded correct responses than to punished reversal errors, whereas offenders with antisocial personality disorder and psychopathy showed significantly less activity. These results augment those from previous studies that have suggested structural ${ }^{29}$ and functional ${ }^{37}$ abnormalities within the superior temporal gyrus in individuals with psychopathy. Moreover, they suggest that this dysfunction contributes to deficits in decision making. ${ }^{36}$

This study has several limitations. The violent offenders with antisocial personality disorder who participated in the study, like most men with this disorder, had additional personality disorders and current or previous substance use disorders. The proportions of such disorders that could affect reversal learning, however, were similar in our two offender groups. The observed functional differences, therefore, cannot simply be attributed to these factors. The use of the contrast of punished reversal errors versus rewarded correct responses cannot distinguish between value representations (contingency tracking) and prediction errors (the amount and valence of surprise associated with feedback on a given trial). The strengths of the study included diagnoses and PCL-R ratings made by trained clinicians, the use of official criminal records to classify participants, and measurement of substance use before the scan.

We identified neural dysfunctions in violent offenders with antisocial personality disorder and psychopathy that 
did not characterise antisocial personality disorder in violent offenders without psychopathy or non-offenders. The important areas of differential activity in offenders with antisocial personality disorder and psychopathy were the posterior cingulate cortex and anterior insula, where activity was increased in response to punished reversal errors, which is indicative of dysfunctional prediction error signalling. Additionally, offenders in this group were hyporesponsive to reward information in the superior temporal gyrus, which suggests a failure to consolidate reward information. We have provided further evidence of distinctive neural anomalies that distinguish between individuals with antisocial personality disorder with and without psychopathy. Diagnostic classification schemes, offender rehabilitation programmes, ${ }^{38}$ and childhood prevention programmes would benefit from taking account of this mounting evidence.

\section{Contributors}

RJB, Dff, VK, SH, and NB conceived and designed the study and Dff, VK, SH, and NB obtained funding. SG and NB were responsible for acquisition of data. SG, RJB, Dff, and NB analysed and interpreted the data. SG, SH, and NB did the statistical analyses. AS, SH, and NB provided administrative, technical, or material support. SH and NB supervised the study. SG, SH, and NB drafted the paper, and all authors contributed to critical revision for intellectual content.

\section{Declaration of interests}

We declare no competing interests.

References

1 Moffitt TE, Caspi A, Harrington H, Milne BJ. Males on the lifecourse-persistent and adolescence-limited antisocial pathways: follow-up at age 26 years. Dev Psychopathol 2002; 14: 179-207.

2 Hare RD, Hart SD, Harpur TJ. Psychopathy and the DSM-IV criteria for antisocial personality disorder. J Abnorm Psychol 1991; 100: 391-98.

3 Dawel A, O'Kearney R, McKone E, Palermo R. Not just fear and sadness: meta-analytic evidence of pervasive emotion recognition deficits for facial and vocal expressions in psychopathy. Neurosci Biobehav Rev 2012; 36: 2288-304.

4 Kosson DS, Lorenz AR, Newman JP. Effects of comorbid psychopathy on criminal offending and emotion processing in male offenders with antisocial personality disorder. J Abnorm Psychol 2006; 115: 798-806.

5 Frick PJ, Ray JV, Thornton LC, Kahn RE. Can callous-unemotional traits enhance the understanding, diagnosis, and treatment of serious conduct problems in children and adolescents? A comprehensive review. Psychol Bull 2014; 140: 1-57.

6 Guy LS, Edens JF, Anthony C, Douglas KS. Does psychopathy predict institutional misconduct among adults? A meta-analytic investigation. J Consult Clin Psychol 2005; 73: 1056-64.

7 Gregory S, Ffytche D, Simmons A, et al. The antisocial brain: psychopathy matters. Arch Gen Psychiatry 2012; 69: 962-72.

8 Decety J, Skelly LR, Kiehl KA. Brain response to empathy-eliciting scenarios involving pain in incarcerated individuals with psychopathy. JAMA Psychiatry 2013; 70: 638-45.

9 Verona E, Sprague J, Sadeh N. Inhibitory control and negative emotional processing in psychopathy and antisocial personality disorder. J Abnorm Psychol 2012; 121: 498-510.

10 Rangel A, Camerer C, Montague PR. A framework for studying the neurobiology of value-based decision making. Nat Rev Neurosci 2008; 9: 545-56.

11 Budhani S, Richell RA, Blair RJ. Impaired reversal but intact acquisition: probabilistic response reversal deficits in adult individuals with psychopathy. J Abnorm Psychol 2006; 115: 552-58.

12 De Brito SA, Viding E, Kumari V, Blackwood N, Hodgins S. Cool and hot executive function impairments in violent offenders with antisocial personality disorder with and without psychopathy. PLoS One 2013; 8: e65566.

13 Hare TA, Camerer CF, Rangel A. Self-control in decision-making involves modulation of the vmPFC valuation system. Science 2009; 324: $646-48$
14 Newman JP, Kosson DS. Passive avoidance learning in psychopathic and nonpsychopathic offenders. J Abnorm Psychol 1986; 95: 252-56.

15 Newman JP, Patterson CM, Kosson DS. Response perseveration in psychopaths. J Abnorm Psychol 1987; 96: 145-48.

16 Brazil IA, Maes JH, Scheper I, et al. Reversal deficits in individuals with psychopathy in explicit but not implicit learning conditions. J Psychiatry Neurosci 2013; 38: E13-20.

17 von Borries AK, Brazil IA, Bulten BH, Buitelaar JK, Verkes RJ, de Bruijn ER. Neural correlates of error-related learning deficits in individuals with psychopathy. Psychol Med 2010; 40: 1559-68.

18 Blair RJ, Peschardt KS, Budhani S, Mitchell DG, Pine DS. The development of psychopathy. J Child Psychol Psychiatry 2006; 47: 262-76.

19 Remijnse PL, Nielen MM, Uylings HB, Veltman DJ. Neural correlates of a reversal learning task with an affectively neutral baseline: an event-related fMRI study. Neuroimage 2005; 26: 609-18.

20 Budhani S, Marsh AA, Pine DS, Blair RJ. Neural correlates of response reversal: considering acquisition. Neuroimage 2007; 34: 1754-65.

21 Finger EC, Marsh AA, Mitchell DG, et al. Abnormal ventromedia prefrontal cortex function in children with psychopathic traits during reversal learning. Arch Gen Psychiatry 2008; 65: 586-94.

22 Murphy K, Garavan H. Artifactual fMRI group and condition differences driven by performance confounds. Neuroimage 2004; 21: 219-28.

23 Cooke DJ, Michie C. Psychopathy across cultures: North America and Scotland compared. J Abnorm Psychol 1999; 108: 58-68.

24 Budhani S, Blair RJ. Response reversal and children with psychopathic tendencies: success is a function of salience of contingency change. J Child Psychol Psychiatry 2005; 46: 972-81.

25 Pearson JM, Heilbronner SR, Barack DL, Hayden BY, Platt ML Posterior cingulate cortex: adapting behavior to a changing world. Trends Cogn Sci 2011; 15: 143-51.

26 Liu X, Hairston J, Schrier M, Fan J. Common and distinct networks underlying reward valence and processing stages: a meta-analysis of functional neuroimaging studies. Neurosci Biobehav Rev 2011; 35: $1219-36$.

27 Kable JW, Glimcher PW. The neural correlates of subjective value during intertemporal choice. Nat Neurosci 2007; 10: 1625-33.

28 Rijsdijsk FV, Viding E, De Brito S, et al. Heritable variations in gray matter concentration as a potential endophenotype for psychopathic traits. Arch Gen Psychiatry 2010; 67: 406-13.

29 Ermer E, Cope LM, Nyalakanti PK, Calhoun VD, Kiehl KA. Aberrant paralimbic gray matter in criminal psychopathy. J Abnorm Psychol 2012; 121: 649-58.

30 Birbaumer N, Veit R, Lotze M, et al. Deficient fear conditioning in psychopathy: a functional magnetic resonance imaging study. Arch Gen Psychiatry 2005; 62: 799-805.

31 Glenn AL, Raine A, Schug RA. The neural correlates of moral decision-making in psychopathy. Mol Psychiatry 2009; 14: 5-6.

32 Sethi A, Gregory S, Dell'Acqua F, et al. Emotional detachment in psychopathy: involvement of dorsal default-mode connections. Cortex 2014; published online Aug 12. DOI:10.1016/j.cortex.2014.07.018

33 Blair RJ. The amygdala and ventromedial prefrontal cortex in morality and psychopathy. Trends Cogn Sci 2007; 11: 387-92.

34 Klein TA, Ullsperger M, Danielmeier C. Error awareness and the insula: links to neurological and psychiatric diseases. Front Hum Neurosci 2013; 7: 14.

35 Clark L, Bechara A, Damasio H, Aitken MR, Sahakian BJ, Robbins TW. Differential effects of insular and ventromedial prefrontal cortex lesions on risky decision-making. Brain 2008; 131: 1311-22.

36 Hare TA, Camerer CF, Knoepfle DT, Rangel A. Value computations in ventral medial prefrontal cortex during charitable decision making incorporate input from regions involved in social cognition. J Neurosci 2010; 30: 583-90.

37 Harenski CL, Harenski KA, Shane MS, Kiehl KA. Aberrant neural processing of moral violations in criminal psychopaths. J Abnorm Psychol 2010; 119: 863-74.

38 Baskin-Sommers AR, Curtin JJ, Newman JP. Altering the cognitiveaffective dysfunctions of psychopathic and externalizing offender subtypes with cognitive remediation. Clin Psychol Sci (in press). 